\title{
Crusius, Gabriele, Aufklärung und Bibliophilie. Der Hannoveraner Sammler Georg Friedrich Brandes und seine Bibliothek
}

Anne Saada

\section{OpenEdition}

Édition électronique

URL : http://journals.openedition.org/ifha/6639

DOI : $10.4000 /$ ifha.6639

ISSN : 2198-8943

\section{Éditeur}

IFRA - Institut franco-allemand (sciences historiques et sociales)

\section{Référence électronique}

Anne Saada, "Crusius, Gabriele, Aufklärung und Bibliophilie. Der Hannoveraner Sammler Georg Friedrich Brandes und seine Bibliothek », Revue de l'IFHA [En ligne], Date de recension, mis en ligne le 01 janvier 2011, consulté le 22 septembre 2020. URL : http://journals.openedition.org/ifha/6639 ; DOI : https:// doi.org/10.4000/ifha.6639

Ce document a été généré automatiquement le 22 septembre 2020.

(CIFHA 


\title{
Crusius, Gabriele, Aufklärung und Bibliophilie. Der Hannoveraner Sammler Georg Friedrich Brandes und seine Bibliothek
}

\author{
Anne Saada
}

1 Comment écrire l'histoire d'une bibliothèque privée quand l'on possède peu de sources à son sujet ? Tel est l'un des problèmes classiques que rencontre l'histoire du livre, qui n'a souvent affaire qu'à des catalogues de bibliothèques dont l'on ne peut même pas toujours savoir s'ils répertorient les ouvrages d'un ou de plusieurs propriétaires. La reconstitution que propose l'auteur du présent ouvrage - à l'origine, une thèse - est celle de la bibliothèque d'un savant et administrateur important mais peu connu du XVIIIe siècle, Georg Friedrich Brandes (1719-1791). Brandes appartenait au « patriciat d'État » (Joachim Campe) du Hanovre, notion qui désigne l'élite au service de l'État, de l'administration, qu'elle appartienne ou non à la nouvelle noblesse. Concernant Hanovre, il faut savoir que cette ville, capitale de l'électorat, avait pour particularité d'être une ville de résidence sans cour, depuis le départ de celle-ci pour l'Angleterre en 1714, lorsque l'électeur du Hanovre était devenu roi d'Angleterre (Georges Ier). Brandes s'est surtout construit une réputation par les fonctions qu'il a exercées au ministère à Hanovre au sein du département en charge de l'université de Göttingen et qui l'ont amené à jouer un rôle de premier plan pour l'organisation de la bibliothèque universitaire entre 1769 et 1791 . Ensemble, avec Christian Gottlob Heyne, nommé professeur à l'université de Göttingen en 1763 et premier bibliothécaire, ils gouvernèrent l'institution, l'un à partir de Hanovre et l'autre en étant sur place, à Göttingen.

La raison pour laquelle G.C. s'intéresse à Brandes est qu'il était collectionneur d'œuvres d'art et de livres. La collection d'œuvres d'art a malheureusement disparu, ce qui explique que G.C. se concentre sur la bibliothèque de Brandes. Celle-ci fut transférée en entier à Oldenbourg lorsque le duc Peter Friedrich Ludwig en fit l'acquisition en 1789 
dans le but de fonder une bibliothèque publique. Elle contenait alors 21387 volumes. $\mathrm{Au}$ cours du XIXe siècle, la bibliothèque de Brandes n'a pas été conservée comme telle, et les livres de l'administrateur ont été mêlés aux acquisitions du duc. La bibliothèque de Brandes constitue aujourd'hui le noyau dur du fonds ancien de la bibliothèque régionale d'oldenbourg (Landesbibliothek Oldenburg). Les ouvrages ayant appartenu à Brandes sont repérables par leur reliure ainsi que par les ex-libris figurant dans les volumes. Si la bibliothèque n'a pas été conservée en son état originel, on possède par chance le catalogue systématique établi par Brandes lui-même. La source principale utilisée par G.C. pour éclairer l'histoire de la bibliothèque de Brandes est la correspondance de celui-ci et, particulièrement, celle qu'il noua à partir de 1764 avec Christian Gottlob Heyne. Entre octobre 1764 et septembre 1791, Brandes écrivit 1500 lettres à Heyne. Les liens entre les deux hommes, s'ils étaient de nature professionnelle au début, se transformèrent rapidement en relation amicale, puis familiale, par le mariage de la fille cadette de Brandes avec Heyne en secondes noces, en 1777.

C'est par l'entremise de cette correspondance que l'on peut identifier certains des libraires mobilisés par Brandes pour enrichir sa bibliothèque. Est-ce Brandes qui fit profiter Heyne de ses contacts pour l'acquisition des livres à l'échelle européenne comme le pense G.C. - ou à l'inverse, est-ce Heyne qui mit à disposition de Brandes les réseaux de la bibliothèque - comme le suggèrent les registres d'acquisition conservées à la bibliothèque de Göttingen dans lesquels apparaissent des listes intitulées "pour Brandes " ? La réponse se situe certainement entre les deux, et il est probable que les deux hommes aient monté conjointement leurs réseaux d'acquisition. Tous deux avaient recours au libraire Dieterich implanté à Göttingen, à la firme Bauer et Treuttel installée à Strasbourg ainsi qu'à Reich à Leipzig. Une partie importante de la collection de Brandes - la moitié selon G.C. - comme souvent à l'époque, provenait de la vente de catalogues de bibliothèques privées.

4 C'est à partir du catalogue systématique de la bibliothèque que G.C. entreprend une étude de celle-ci. Ce catalogue était divisé en 13 rubriques, elles-mêmes divisées en trois catégories selon les formats : théologie ( $8,6 \%$ de la bibliothèque), jurisprudence $(4,3 \%)$, médecine $(1,4 \%)$, philosophie, auteurs anciens avec leurs commentateurs $(8 \%)$, histoire $(28,4 \%)$, antiquités et mythologie, beaux-arts, arts agréables et utiles, belles lettres $(8,6 \%)$, traités de l'éducation et instruction littéraire, histoire littéraire (3000 volumes), polygraphes et recueils sur différentes matières. À côté de chaque notice bibliographique, Brandes donnait en français des précisions sur la valeur des livres, leur prix, leur rareté, la qualité de l'édition, la reliure, les illustrations, etc. Si la situation financière de Brandes est mal connue, l'on sait en revanche que ses dépenses pour les livres dépassaient de loin ses revenus. On estime que pour la constitution de sa bibliothèque, Brandes dépensa environ 40000 Reichsthaler.

Malgré la correspondance entre Brandes et Heyne, il faut bien l'avouer, rares sont les éléments qui permettent de retracer la constitution de la bibliothèque de Brandes. De même, on dispose de très peu de données sur les usages qui ont été faits de celle-ci : en dehors de quelques visiteurs ou voyageurs identifiés comme Heyne, certains professeurs de l'université de Göttingen, Herder et Klopstock, on ne sait pas si Brandes ouvrait sa collection à des lecteurs, et les indices sur Brandes lecteur sont peu nombreux, en dehors de jugements qu'il a communiqués à son ami Heyne sur certains ouvrages. Au regard du manque de sources, peut-être faut-il considérer, avec G.C., que l'apport principal du catalogue de la bibliothèque de Brandes, est de nous livrer une 
image, à une époque donnée, de ce que contenait la bibliothèque non pas d'un savant ni d'un auteur, mais de celle d'un amateur éclairé, potentiellement représentatif de ce " patriciat d'État » du Hanovre.

6 Anne Saada (Centre national de la recherche scientifique, Paris) 